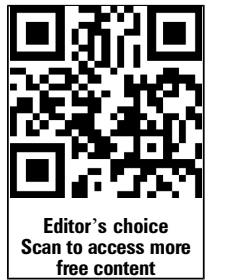

${ }^{1}$ Molecular Pharmacology and Pathology Program, Department of Pathology and Bosch Institute, University of Sydney, Sydney, New South Wales, Australia

2Discipline of Pathology, and Molecular Medicine Research Group, School of Medicine, University of Western Sydney, Sydney, New South Wales, Australia

${ }^{3}$ Cancer Pathology Bosch Institute, University of Sydney, Sydney, New South Wales, Australia

${ }^{4}$ Cancer Pathology \& Cell Biology Laboratory, Ingham Institute for Applied Medical Research, Sydney, New South Wales, Australia

\section{Correspondence to}

Dr Sumit Sahni or

Prof Des R Richardson,

Molecular Pharmacology and Pathology Program,

Department of Pathology and Bosch Institute, University of Sydney, Blackburn Building (D06), Sydney, New South Wales 2006, Australia; sumit.sahni@sydney.edu.au or d.richardson@sydney.edu.au

SS and DRR contributed equally to the work as co-corresponding and senior authors.

Received 14 May 2013 Accepted 15 May 2013 Published Online First 8 June 2013

\title{
The role of NDRG1 in the pathology and potential treatment of human cancers
}

\author{
Dong-Hun Bae, ${ }^{1}$ Patric J Jansson, ${ }^{1}$ Michael L Huang, ${ }^{1}$ Zaklina Kovacevic, ${ }^{1}$ \\ Danuta Kalinowski, ${ }^{1}$ C Soon Lee, ${ }^{2,3,4}$ Sumit Sahni, ${ }^{1}$ Des R Richardson ${ }^{1}$
}

\section{ABSTRACT}

$\mathrm{N}$-myc downstream regulated gene 1 (NDRG1) has been well characterised to act as a metastatic suppressor in a number of human cancers. It has also been implicated to have a significant function in a number of physiological processes such as cellular differentiation and cell cycle. In this review, we discuss the role of NDRG1 in cancer pathology. NDRG1 was observed to be downregulated in the majority of cancers. Moreover, the expression of NDRG1 was found to be significantly lower in neoplastic tissues as compared with normal tissues. The most important function of NDRG1 in inhibiting tumour progression is associated with its ability to suppress metastasis. However, it has also been shown to have important effects on other stages of cancer progression (primary tumour growth and angiogenesis). Recently, novel iron chelators with selective antitumour activity (ie, Dp44mT, DpC) were shown to upregulate NDRG1 in cancer cells. Moreover, Dp44mT showed its antimetastatic potential only in cells expressing NDRG1, making this protein an important therapeutic target for cancer chemotherapy. This observation has led to increased interest in the examination of these novel anticancer agents.

\section{INTRODUCTION}

$\mathrm{N}$-myc downstream regulated gene 1 (NDRG1) (also known as Drg1, RTP, Rit42, PROXY-1 or Cap43) has been well described as a metastasis suppressor in a number of cancers including colon, prostate and breast cancers. ${ }^{1-3}$ The NDRG1 gene is a member of the human NDRG family, which also comprises NDRG2, NDRG3 and NDRG4. ${ }^{4-7}$ Chromosome mapping studies have demonstrated that the NDRG1 gene is located on chromosome eight and encodes a $3 \mathrm{~kb}$ mRNA that is translated into a $43 \mathrm{kDa}$ protein. ${ }^{1489}$ The NDRG family of proteins belongs to the $\alpha / \beta$ hydrolase group of enzymes, although it is notable that the NDRG1 protein lacks a hydrolytic catalytic site and is deficient in hydrolytic enzyme activity. ${ }^{10-12}$ This observation suggests that the gene has been modified by convergent evolution to obtain the same fold for a specific purpose.

NDRG1 is a phosphorylated protein that contains five calmodulin kinase 2 phosphorylation sites, three serine phosphorylation sites, a casein kinase II site, five myristoylation sites, three protein kinase $\mathrm{C}$ phosphorylation sites, one thioesterase site, one tyrosine phosphorylation site and one phosphopantotheine attachment site. ${ }^{13}$ Furthermore, NDRG1 has been shown to be phosphorylated by upstream kinases like serum/glucocorticoid regulated kinase 1 and glycogen synthase kinase 3 (which are essential Ser/Thr kinase family proteins). ${ }^{14}$ The role of phosphorylation at numerous sites on the NDRG1 protein is still unknown, but may be related to the numerous physiological functions of NDRG1. ${ }^{15}$ The $\mathrm{N}$-terminus of NDRG1 protein consists of two myc boxes (MBI and MBII), which are crucial for the protein's function, while the central region consists of MBIII (involved in cell transformation and apoptosis) and MBIV (for apoptosis induction, transformation and G2 arrest). ${ }^{16-18}$

Of interest, NDRG1 cDNA contains multiple CpG islands at its $5^{\prime}$ end, suggesting that DNA methylation can control NDRG1 expression. ${ }^{19}$ The NDRG1 gene has three hypoxia inducible factor 1 (HIF-1) binding sites, with one situated in its promoter and the remaining two in its $3^{\prime}$ untranslated region,${ }^{20}$ indicating that NDRG1 may be regulated by HIF-1 through its binding sites in the untranslated region. ${ }^{21}$

\section{DISTRIBUTION OF NDRG1 IN CELLS AND TISSUES}

The expression of NDRG1 mRNA is ubiquitous among human tissues, but higher levels are found in the prostate, brain, kidney, placenta and intestinal tissues. ${ }^{1}{ }^{4}$ However, the NDRG1 protein is mainly found in the epithelium, which suggests that it may have a specific function related to these types of cells. ${ }^{22}$

At the cellular level, the NDRG1 protein is predominantly cytoplasmic in nature. ${ }^{22}$ However, this localisation can vary between different cell types (eg, intestinal and breast epithelia express membrane-associated protein, whereas prostate epithelial cells demonstrate nuclear localisation of NDRG1 ${ }^{22}$ ). In some cell types, mitochondrial localisation is also observed..$^{22}$ Collectively, these findings suggest that NDRG1 functions in a tissue-specific manner. ${ }^{1}$ However, some studies have observed that NDRG1 distribution is not tissue-specific. ${ }^{4}{ }^{23}$ Moreover, analysis using PSORTII software also predicts that NDRG1 is primarily a cytoplasmic protein $(47.8 \%)$, followed by its localisation in the nucleus (26.1\%) and mitochondrion (8.7\%).

Membrane-associated NDRG1 protein has been mostly found adjacent to adherens junctions, where intermediate and microfilament bundles insert into these structures. ${ }^{22}$ This finding is suggestive of the involvement of NDRG1 in cell adhesion and this may be important for its ability to upregulate the levels of E-cadherin that plays a crucial role in forming the adherens complex. ${ }^{22}$

\section{FUNCTION OF NDRG1 AND ITS REGULATION NDRG1 and cellular differentiation}

The exact function(s) of NDRG1 still remain elusive, but numerous recent studies have suggested 
that this molecule is involved in cellular differentiation (see figure 1). ${ }^{8}{ }^{24}$ Using a variety of cell types, including keratinocytes, U937 myelomonocytic cells, colonic cancer cells, mast cells, renal cells, monocytes/macrophages and prostate cancer cells, revealed that differentiation positively correlated with increased NDRG1 expression induced by peroxisomal proliferator-activated receptor $\gamma .^{25-28}$ The role of NDRG1 in differentiation was further illustrated in experiments performed on rat peripheral glioma cells (D6P2T), which were induced to differentiate by the cAMP phosphodiesterase inhibitor isobutylmethyl xanthine. ${ }^{29}$ This treatment resulted in a cessation of proliferation which coincided with a significant increase in NDRG1. ${ }^{29}$

Of interest, genes which are known to suppress cellular differentiation (eg, Hod, S100a10, connective tissue growth factor, ectonucleotide pyrophosphatase/phosphodiesterase family member 3 and secreted phosphoprotein 1) were shown to be downregulated by NDRG1, resulting in increased cellular differentiation. ${ }^{8}$ In addition, NDRG1 induces Wnt suppression which restores the epithelial cell-cell adhesion complex (E-cadherin$\beta$-catenin). ${ }^{30}$ This is crucial for maintaining epithelial cell morphology, polarity and motility ${ }^{30}$ that is linked to a differentiated phenotype. The effect of NDRG1 on differentiation is probably linked to its effects on a variety of other molecular targets and these are described below.

\section{NDRG1 and cell cycle control}

Studies have noted that the expression of NDRG1 is biphasic throughout the cell cycle, peaking during the G1 and G2/M phases and decreasing to its lowest level during $S$ phase. ${ }^{31}$ This finding indicates a potential role in G0/G1 arrest potentially through altered expression of p21WAF1/cip1 and cyclindependent kinase 1 and $4 .{ }^{31} 32$ Interestingly, NDRG1 was found to upregulate $\mathrm{p} 21 \mathrm{WAF} 1 / \mathrm{cip} 1$ protein levels in prostate and lung cancer cells. ${ }^{33}$ However, NDRG1 expression had no effect on proliferation or the cell cycle of these latter cells; rather, it was able to inhibit cell migration. ${ }^{33}$ Potentially, this may indicate a cell type dependent response.

NDRG1 is also a microtubule-associated protein mainly located in centromeres that could be involved in p53-dependent spindle checkpoint and in mitosis. ${ }^{134}$ The main evidence for the role of NDRG1 in its role in the maintenance of euploidy is derived from studies performed using p53-negative cancer cell lines. ${ }^{35}$ When these cells were exposed to the microtubule inhibitor, taxol, NDRG1 expression was induced and the population of cells in $\mathrm{M}$ phase of the cell cycle was increased. ${ }^{35}$

Notably, phosphorylated NDRG1 has been found at the ends of microtubule bundles during late telophase. ${ }^{36}$ This observation suggests that NDRG1 could be involved in the attachment of mitotic spindles at the point of abscission, as well as cytokinesis regulation. ${ }^{36}$ Overexpression of NDRG1 has also been demonstrated to decrease expression of the Wnt-responsive gene, cyclin D1, which would inhibit cell cycle progression. ${ }^{37}$ In summary, NDRG1 appears to have a variety of effects on cell cycle control molecules that appear in some cases to be cell type-dependent.

\section{NDRG1 and the stress response}

High levels of NDRG1 usually reflect exposure of cells to conditions inducing stress. ${ }^{32}$ Expression of NDRG1 is induced by a range of stress inducing stimuli such as hypoxia, homocysteine, nickel, androgens, calcium and iron depletion. ${ }^{38-40}$ Hypoxia is an essential factor in solid tumour formation and is known to induce the generation of mitochondrial reactive oxygen species. ${ }^{41}$ It also activates HIF-1 $\alpha$, EGR1, nuclear factor- $\kappa \mathrm{B}$ and other transcription factors involved in tumour angiogenesis and invasion. ${ }^{41-43}$ The HIF-1 transcription factor permits quick adaptation and survival when cells are exposed to reduced
Figure 1 Regulation and functions of NDRG1 in cancer. The oncogenes, c-Myc and N-Myc, are often found to be upregulated in cancers and are known to suppress NDRG1. NDRG1 is upregulated by transcription factors HIF-1 and AP1, which are known to be deregulated in some tumours. NDRG1 is upregulated by p53, an apoptotic protein known to be mutated or reduced in variety of cancers. The tumour suppressor, phosphatase and tensin homologue (PTEN), and stress response gene, EIF3a, are also shown to positively regulate NDRG1 expression in cells. NDRG1 can promote cellular differentiation leading to suppression of tumour progression. NDRG1 suppresses angiogenesis via suppression of vascular endothelial growth factor (VEGF) and IL-8. It also mediates suppression of cellular proliferation. NDRG1 inhibits metastatic progression of cancer cells via inhibition of epithelial to mesemchymal transition (EMT).

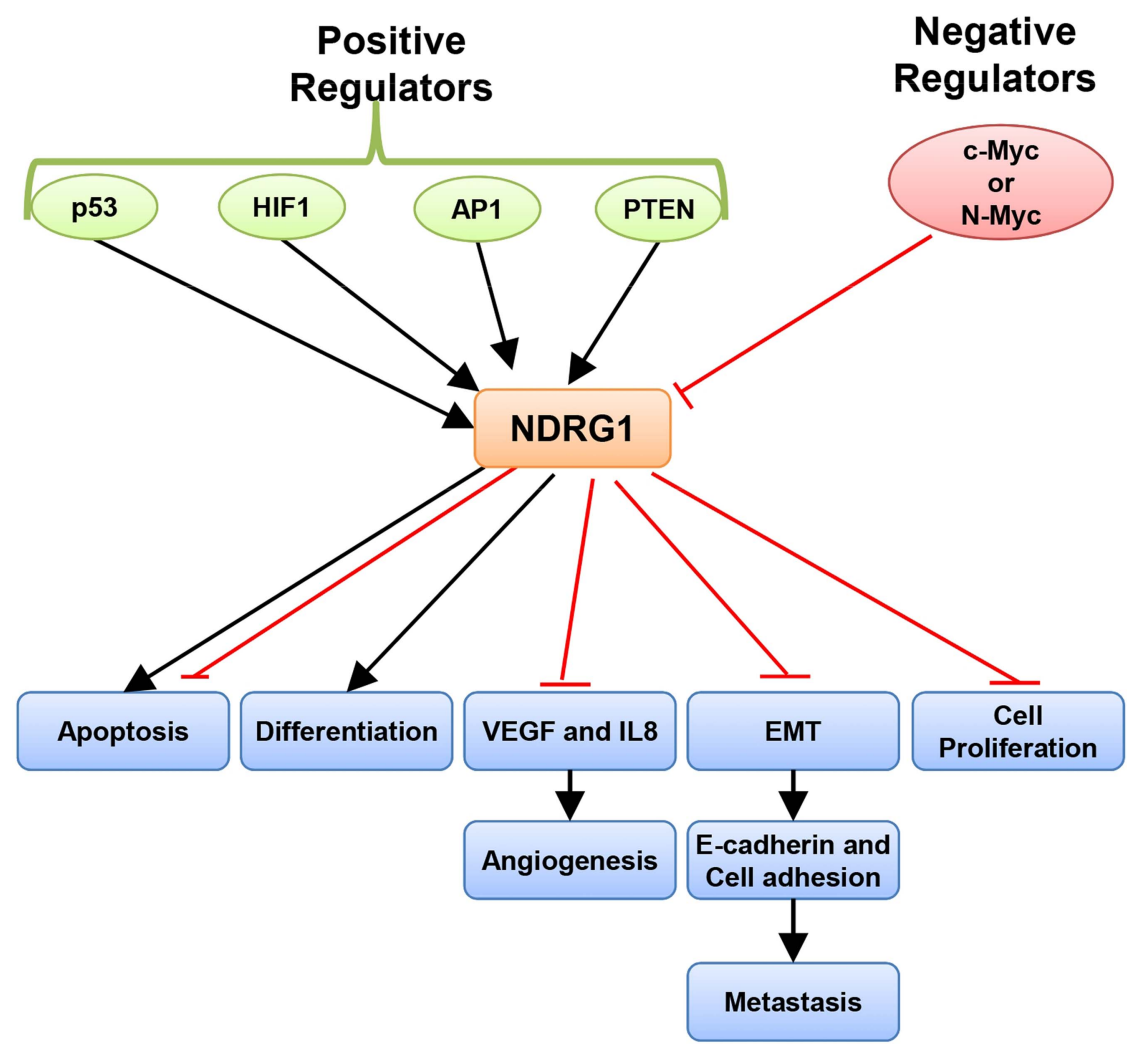


oxygen concentrations ${ }^{44}$ and plays an essential role in regulating hypoxia-responsive genes including NDRG1 (see figure 1). ${ }^{64} 46$ Studies have shown that NDRG1 upregulation can occur via HIF-1 $\alpha$-dependent and -independent mechanisms. ${ }^{46-48}$ In addition, NDRG1 was demonstrated to be regulated by Erg-1 under hypoxic conditions. $^{42}$

Eukaryotic initiation factor $3 a$ (eIF3a) is believed to be a constituent of stress granules, which are intracellular complexes formed on exposure to stressors such as hypoxia and oxidative stress. ${ }^{49}$ These granules act as triage centres, which selectively allow translation of proteins essential for the stress response. ${ }^{50}$ A recent study has shown that eIF3a positively regulated the expression of NDRG1 under iron-depleted conditions. ${ }^{40}$ This again re-emphasises the role of NDRG1 as an important stress response protein.

\section{NDRG1 and PTEN}

The NDRG1 protein is involved in regulating the negative feedback-loop linking the phosphoinositide 3-kinase (PI3K) pathway and the tumour suppressor, phosphatase and tensin homologue (PTEN). ${ }^{26}$ This observation was first noted in prostate cancer cells where PTEN expression is often lost due to low NDRG1 levels, which results in increased disease progression. ${ }^{26}$ In fact, when PTEN levels were upregulated in prostate and breast cancer cells, NDRG1 expression was also increased in a dose- and time-dependent manner. ${ }^{51}$ On the other hand, inhibiting PTEN expression using siRNA led to a reduction in NDRG1 levels. ${ }^{51}$ Interestingly, recent studies demonstrated that NDRG1 can also upregulate PTEN protein levels in pancreatic cancer cells. ${ }^{26}$ Hence, these latter molecules positively regulate each other.

In cancer cells, the balance in the PI3K/PTEN feedback-loop is frequently lost, which leads to increased PI3K signalling and reduced PTEN levels. ${ }^{26}$ This feedback-loop can be rebalanced by upregulating NDRG1, which subsequently increases PTEN expression, leading to inhibition of PI3K signalling and phosphorylated protein kinase B (pAKT), halting tumour progression. $^{2652}$

\section{NDRG1 and p53}

The tumour suppressor gene, p53, which induces NDRG1 in a cell type-specific manner, may control cell proliferation, caspase activation and apoptosis (see figure 1). ${ }^{38}{ }^{39}$ Activation of NDRG1 occurs as a result of p53 binding to its promoter. ${ }^{38}$ This was revealed in the colon cancer cell line (DLD-1), where NDRG1 was upregulated after p53 induction. ${ }^{38}$ It was also shown that the expression of NDRG1 is induced by DNA damaging agents in a process which is dependent on p53. ${ }^{38}$ Moreover, it was also observed that NDRG1 was required for the induction of p53-mediated apoptosis in the colon cancer cell line, DLD-1. ${ }^{38}$ Conversely, other studies involving lung cancer cell lines have shown a lack of correlation between NDRG1 expression and DNA damage even though p53 is upregulated. $^{29} 46$ These observations potentially indicate cell- and tissue-specific regulation of NDRG1 via p53.

In addition, EGR1 can upregulate p53 which can, in turn, positively regulate both $\mathrm{p} 21 \mathrm{WAF} 1 / \mathrm{cip} 1$ and NDRG1. ${ }^{12} 52-54$ Moreover, as mentioned earlier, NDRG1 itself can also upregulate p21WAF1/cip1. ${ }^{33}$ Subsequently, p21WAF1/cip1 inhibits cell cycle progression and promotes apoptosis by inducing G1/S arrest. ${ }^{33}$ This latter molecule can also inhibit cell migration and metastasis of cancers. ${ }^{33}$

\section{NDRG1 and MYC}

Overexpression of N-myc and c-myc genes represses NDRG1 expression by binding to the $\mathrm{N}$-myc binding motif close to the initiation promoter (see figure 1). ${ }^{29} 56$ This was shown in mice where the mouse homologue of NDRG1 (Ndr-1) was transcriptionally repressed by the N-myc:Max heterodimer, which binds to the Ndr-1 promoter region. ${ }^{57}$ Moreover, in IMR-32 and NGP neuroblastoma cells, N-myc suppression by retinoids promoted NDRG1 expression. ${ }^{29}$ As a result, retinoids can induce cellular differentiation, which could increase NDRG1 levels by other pathways. ${ }^{29}$ Interestingly, nickel upregulates NDRG1 in some cell types and also inhibits $\mathrm{N}$-myc expression. ${ }^{29}$

\section{ROLE OF NDRG1 IN PRIMARY TUMOUR GROWTH, METASTASIS AND ANGIOGENESIS}

It is notable that NDRG1 is widely expressed in normal cells and tissues. ${ }^{22}$ In contrast, NDRG1 was observed to be downregulated in the majority of cancers. ${ }^{2}$ A range of prostate cancer specimens analysed by immunohistochemistry have shown that NDRG1 expression is lower in neoplastic when compared with adjacent normal tissues. ${ }^{19}$ This finding was further substantiated by the discovery of an inverse relationship between the Gleason grade of the cancer with NDRG1 expression levels, where a higher grade corresponds to poorly differentiated tumours with low NDRG1 protein expression. ${ }^{58} 59$ This finding is also observed in colon cancer where the expression of NDRG1 declines as normal colonic epithelium progresses to carcinoma. ${ }^{60}$ The decline in NDRG1 expression was revealed to correlate with a shorter 5-year survival rate in colonic cancer patients. $^{60}$

\section{Role of NDRG1 in primary tumour growth}

The NDRG1 protein has a significant role in inhibiting primary tumour growth. Increased expression of NDRG1 resulted in a reduction in tumour microvascular density, invasion depth and histopathological grading. ${ }^{61}$ This molecule also reduced proliferation rates in bladder cancer cell lines by up to $70 \% .{ }^{62}$ The primary growth of the human breast, prostate and bladder cancer cell lines was inhibited by NDRG1, leading to suppression of anchorage-independent growth in soft agar. ${ }^{24}$ In contrast, other studies have shown that NDRG1 overexpression had no effect on pancreatic cancer cell growth in vitro, as well as in colon and prostate tumours. ${ }^{19}{ }^{63}$ In addition, no correlation was observed between size or histological grade of breast cancer and NDRG1 expression. ${ }^{51}$

\section{Role of NDRG1 as a metastasis suppressor}

The NDRG1 gene is likely to be involved in metastasis suppression due to its negative association with tumour metastasis and cell migration. ${ }^{64-67}$ In fact, in vivo studies demonstrated that NDRG1 significantly inhibited the metastasis of rat prostate cancer AT6.1 cells to the lungs. ${ }^{19}$ Further studies subsequently demonstrated that NDRG1 inhibited metastasis by reducing cell-matrix and cell-cell adhesion in the AT6.1 cells in vitro. ${ }^{8}$ Interestingly, in an investigation using breast cancer cells, upregulation of E-cadherin by NDRG1 reduced the motility of these cells (see figure 1). ${ }^{63}$ These studies suggested that NDRG1 modulates important adhesion molecules such as E-cadherin to inhibit metastasis. These early observations were further confirmed by studies demonstrating a significant increase in the adherens junction proteins E-cadherin and $\beta$-catenin at the plasma membrane in response to NDRG1 overexpression. ${ }^{37}$ In fact, while transforming growth factor- $\beta$ (TGF- $\beta$ ) reduced 
E-cadherin and $\beta$-catenin levels in these DU-145 prostate and HCT116 colon cancer cells, NDRG1 was able to significantly inhibit this effect. ${ }^{37}$ On the other hand, silencing NDRG1 using siRNA in these cells led to a marked reduction in E-cadherin and $\beta$-catenin membrane levels. ${ }^{37}$ Moreover, NDRG1 overexpression also markedly inhibited cell migration and invasion in these cells in vitro. ${ }^{37}$ Hence, NDRG1 promotes the formation of the adherens junctions, leading to increased cell-cell adhesion and reduced cell migration and invasion, resulting in reduced metastatic potential.

Interestingly, the NDRG1 protein is involved in different roles in metastatic and non-metastatic cells. ${ }^{49}$ NDRG1 expression inhibited cell proliferation in metastatic (H1299-NDRG1) cell line, but had no effect in non-metastatic (DLD-1-NDRG1) cells. $^{38}$ In human prostate cancer cells, NDRG1 expression levels were much higher in organ-confined tumours than in lymph node or bone metastases. ${ }^{19}$ Thus, NDRG1 can be used as a molecular biological marker for tumour prognosis. ${ }^{6} 65$ This was confirmed in studies showing that NDRG1 may predict early invasion, metastasis and detect hypoxic regions within the tumour mass of gastric cancer cells, as well as determining patient prognosis. ${ }^{6} 65$

In metastatic cancer cells, actin is polymerised to form stress fibres which help in cellular migration and metastasis. ${ }^{68}$ It has been shown that phosphorylation of myosin light chain 2 (pMLC2) by ROCK1 has an important role in the formation of stress fibres (see figure 2). ${ }^{69}$ In a recent study, it has been shown that NDRG1 can inhibit the ROCK1/pMLC2 pathway, leading to suppression of stress fibre assembly and rearrangement. This acts as an additional pathway via which NDRG1 can modulate its antimetastatic effects. ${ }^{70}$

\section{Role of NDRG1 in angiogenesis}

The NDRG1 gene has an important role in regulating angiogenesis (see figure 1). ${ }^{67}$ Interestingly, the mRNA levels of the two angiogenic factors, vascular endothelial growth factor (VEGF-1) and interleukin-8 (IL-8), were reduced in cells when NDRG1 was overexpressed. ${ }^{61}$ Pancreatic cancer cells with high NDRG1 levels had a significant reduction in both VEGF-1 and IL-8 protein, as well as reduced matrix metalloproteinase- 9 activity which leads to modulation of angiogenesis. ${ }^{71-73}$ In addition, NDRG1 expression was negatively correlated to tumour microvascular density via inhibition of nuclear factor- $\kappa \mathrm{B}$, chemokines and VEGF-A. ${ }^{74}$ Conversely, other studies demonstrate that in cervical cancer patients the enhanced tumour expression of NDRG1 correlated with a higher tumour microvessel density. ${ }^{24}$ These differences could again reflect the tissue-specific function of NDRG1.

\section{TARGETING NDRG1 AS A NOVEL ANTIMETASTATIC THERAPY}

The expression of NDRG1 can be markedly increased in multiple cancer types in vitro and in vivo by novel anticancer agents, namely, the thiosemicarbazone iron chelators of the di-2-pyridylketone thiosemicarbazone class, di-2-pyridylketone 4,4-dimethyl-3-thiosemicarbazone (Dp44mT) and di-2-pyridylketone 4-cyclohexyl-4-methyl-3-thiosemicarbazone (DpC; figure 3A). ${ }^{33} 3746$ These compounds are unique new generation ligands that unlike typical chelators (eg, desferrioxamine, deferisirox, deferiprone; figure $3 \mathrm{~B}$ ) do not induce whole body iron depletion at optimal doses. ${ }^{75-77}$ In fact, the mechanism of action of these ligands involves the binding of iron and/ or copper and the formation of a redox active complex that generates cytotoxic reactive oxygen species. ${ }^{78-80}$ This dual activity of these agents has been termed the 'double punch' as they deplete cells of iron that is critical for proliferation, while at the same time forming a redox-active iron/copper complexes that damages cancer cell lysosomes and this induces apoptosis and cell death (figure $3 \mathrm{C})^{78-80}$

Targeting iron in cancer cells is a novel approach to the treatment of this disease. Indeed, iron is an essential element that is necessary for cell proliferation. ${ }^{81}$ Moreover, cancer cells, which proliferate rapidly, have higher requirements for iron than normal cells. ${ }^{82}$ This is reflected by the increased expression
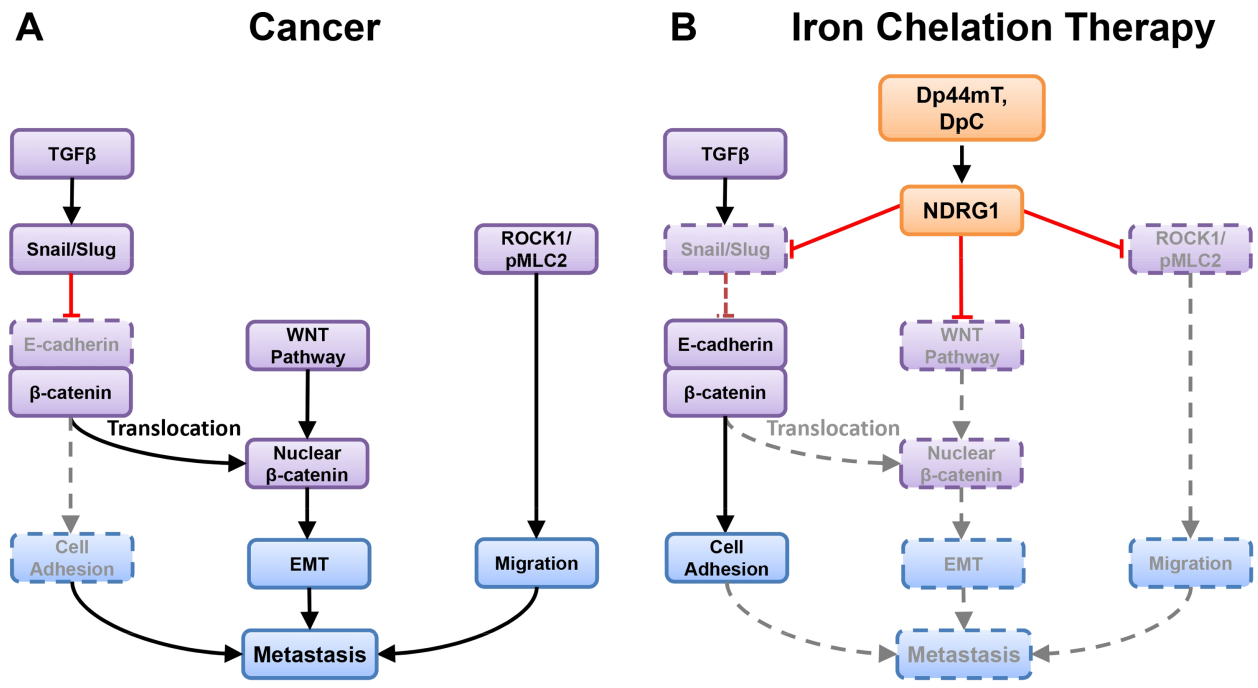

Figure 2 The role of NDRG1 in cancer progression and iron chelation therapy. (A) Cancer pathology. TGF- $\beta$ can promote tumourigenesis via upregulation of Snail/slug pathway. Increased Snail/slug inhibits membrane bound E-cadherin and increases nuclear translocation of $\beta$-catenin. This leads to increased epithelial to mesemchymal transition (EMT) followed by metastasis. Similarly, WNT-signalling pathway can also promote metastasis by increasing the levels of nuclear $\beta$-catenin. ROCK1/pMLC2 modulates actin-filament polymerisation, stress fibre assembly and formation, leading to increased cellular motility and metastasis. (B) Iron chelation chemotherapy. Novel iron chelators (Dp44mT, DpC) upregulate NDRG1 levels, which leads to inhibition of Snail/slug, Wnt-signalling and ROCK1/pMLC2 pathways and, thus, inhibit metastasis. 
Figure 3 Line drawings of the structures of: (A) Dp44mT and DpC; (B) desferrioxamine (DFO), deferisirox (Exjade) and deferiprone.

(C) Schematic illustrating the double punch mechanism that is mediated by thiosemicarbazone chelators of the DpT class. The Dp44mT ligand can bind tumour iron or copper, designated as metal (M) in the schematic. The Dp44mT-M complex ( $\mathrm{M}=\mathrm{Fe}$ (III) or $\mathrm{Cu}(\mathrm{II}))$ can redox cycle upon interaction with cellular reductants (eg, NADH) to generate the reduced complexes, namely, either Dp44mT-Fe(II) complex or Dp44mT-Cu(I) complex. These complexes can subsequently react with oxygen via the Fenton reaction to form reactive oxygen species that mediate oxidative insults to the cell.
A<smiles>CN(C)C(=S)NN=C(c1ccccn1)c1ccccn1</smiles>

Dp44mT<smiles>CN(C(=S)NN=C(c1ccccn1)c1ccccn1)C1CCCCC1</smiles>

DpC
B<smiles>CC(=O)N(O)CCCC(=O)NCCN(O)C(=O)CCNC(=O)CCC(=O)N(CCN)C(=O)O</smiles>

\section{DFO}<smiles>O=C(O)c1ccc(-n2nc(-c3ccccc3O)nc2-c2ccccc2O)cc1</smiles>

Deferisirox<smiles>Cc1c(O)c(=O)ccn1C</smiles>

Deferiprone

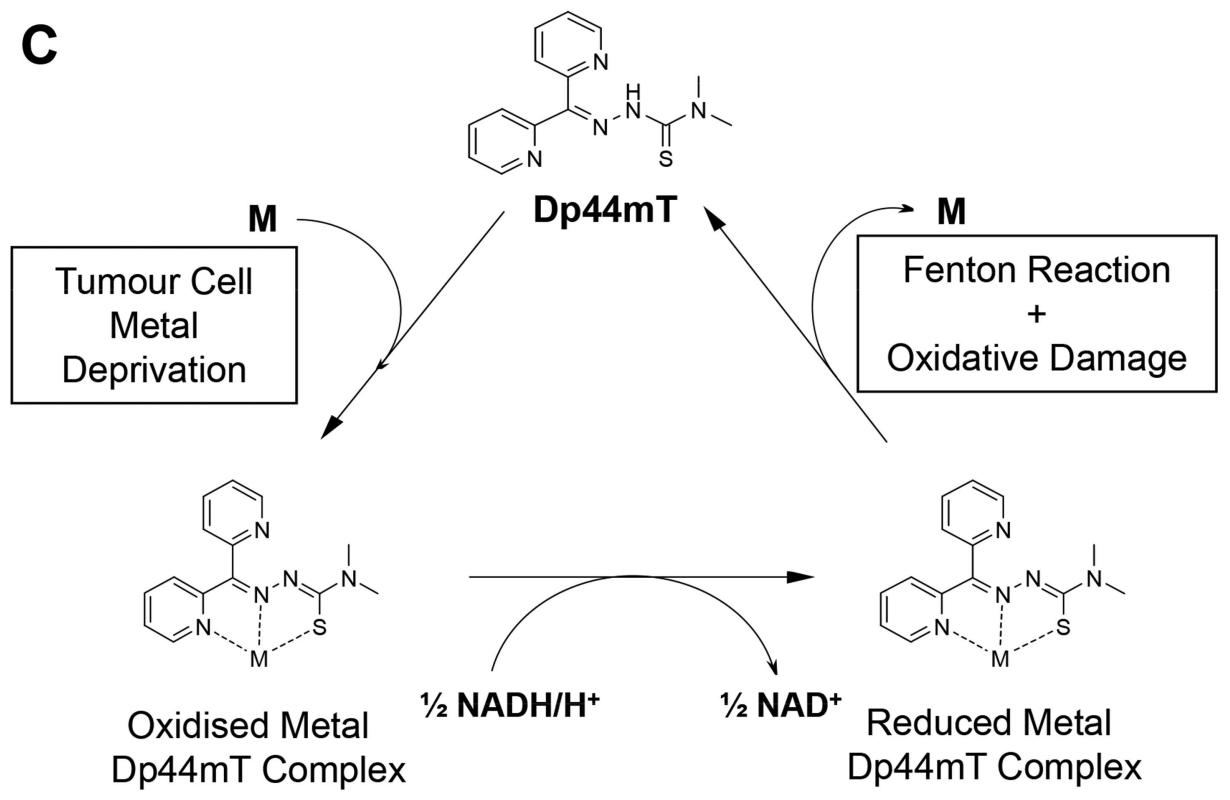

of the transferrin receptor 1 on cancer cells, which is the primary mechanism of iron uptake. ${ }^{82} 83$ Iron is also a critical requirement for many signalling cascades, such as HIF- $1 \alpha,{ }^{84}$ JNK/P38/MAPK, ${ }^{84}$ p53/p21/Cyclin D1 ${ }^{84}$ and Wnt/ß-catenin. ${ }^{85}$ Hence, considering their diverse molecular targets, iron chelators may be a promising new therapeutic approach to reverse or prevent cancer metastasis.

The novel iron chelator, Dp44mT, was able to maintain the expression of epithelial markers, E-cadherin and $\beta$-catenin, attenuating the TGF- $\beta$-induced epithelial to mesemchymal transition in prostate and colorectal cancer cells. ${ }^{37}$ Moreover, this effect was mediated via NDRG1 upregulation and subsequent inhibition of the SMAD/Snail and Wnt pathways (see figure 2). ${ }^{37}$ Furthermore, in our studies, we demonstrate that both Dp44mT and DpC inhibit ROCK1/pMLC2-modulated actinfilament polymerisation, stress fibre assembly and formation via a mechanism involving NDRG1 activation (see figure 2). ${ }^{70} \mathrm{~A}$ recent study showed marked suppression in metastasis upon treatment with Dp44mT in vivo. ${ }^{30}$ Furthermore, these investigators observed a significant reduction in the ability of Dp44mT to inhibit metastasis in xenografts of NDRG1-knockdown
MDA-MB-231-BoM cells, demonstrating the importance of NDRG1 as a therapeutic target in Dp44mT-mediated metastasis suppression. ${ }^{30}$ These results highlight the potential of novel iron chelators as inhibitors of cancer metastasis in tumours that are regulated by NDGR1.

\section{SUMMARY}

NDRG1 has been shown to play an important role in both physiological as well as pathophysiological conditions. It plays a critical role in cancer progression, mainly due to its inhibitory effects on cancer metastasis, via its interaction with key signalling pathways, such as PI3K and WNT. Moreover, novel iron chelators (ie, DpC) have shown to upregulate NDRG1 and have been demonstrated to have potent antitumour activity. This has led to an interest in the use of these novel ligands as potential chemotherapeutics. In conclusion, further elucidation of the molecular mechanisms that underlie the antimetastatic effects of NDRG1 will facilitate the development of new therapies for inhibiting cancer metastasis. 
Key messages

- NDRG1 has important function in cancer pathology, mainly by its ability to inhibit metastasis.

- Novel iron chelators (DpC, Dp44mT) can upregulate NDRG1 expression via hypoxia inducible factor-1 $\alpha$-dependent and independent mechanisms.

- NDRG1 is required for the anti-metastatic activity of Dp44mT.

- Novel iron chelators have shown the potential to be developed as chemotherapeutics against metastatic cancers.

Acknowledgements DRR thanks the National Health and Medical Research Council of Australia (NHMRC) for a Senior Principal Research Fellowship and Project Grant funding. DSK appreciates NHMRC Project Grant support. PJJ and ZK kindly acknowledge the NHMRC, Cancer Institute of New South Wales (CINSW) and/or Prostate Cancer Foundation of Australia for Early Career Research Fellowships.

Contributors All authors contributed to design, writing and presentation of the review.

Funding NHMRC, Australia.

Competing interests None.

Provenance and peer review Not commissioned; internally peer reviewed.

\section{REFERENCES}

1 Kovacevic Z, Richardson DR. The metastasis suppressor, Ndrg-1: a new ally in the fight against cancer. Carcinogenesis 2006;27:2355-66.

2 Shah MA, Kemeny N, Hummer A, et al. Drg1 expression in 131 colorectal liver metastases: correlation with clinical variables and patient outcomes. Clin Cancer Res 2005;11:3296-302.

3 Motwani M, Sirotnak FM, She Y, et al. Drg1, a novel target for modulating sensitivity to CPT-11 in colon cancer cells. Cancer Res 2002;62:3950-5.

4 Ellen TP, Ke Q, Zhang P, et al. NDRG1, a growth and cancer related gene: regulation of gene expression and function in normal and disease states. Carcinogenesis 2008;29:2-8

5 Cheng J, Xie HY, Xu X, et al. NDRG1 as a biomarker for metastasis, recurrence and of poor prognosis in hepatocellular carcinoma. Cancer Lett 2011;310:35-45.

6 Said HM, Stein S, Hagemann C, et al. Oxygen-dependent regulation of NDRG1 in human glioblastoma cells in vitro and in vivo. Oncol Rep 2009;21:237-46.

7 Fotovati A, Abu-Ali S, Sugita Y, et al. Expression of N-myc downstream regulated gene 1 (NDRG1) in central neurocytoma. J Clin Neurosci 2011;18:1383-5.

8 Kovacevic Z, Fu D, Richardson DR. The iron-regulated metastasis suppressor, Ndrg-1: identification of novel molecular targets. Biochim Biophys Acta 2008;1783:1981-92.

9 Zhao G, Chen J, Deng Y, et al. Identification of NDRG1-regulated genes associated with invasive potential in cervical and ovarian cancer cells. Biochem Biophys Res Commun 2011;408:154-9.

10 Hickok JR, Sahni S, Mikhed Y, et al. Nitric oxide suppresses tumor cell migration through N-Myc downstream-regulated gene-1 (NDRG1) expression: role of chelatable iron. J Biol Chem 2011;286:41413-24.

11 King RH, Chandler D, Lopaticki S, et al. Ndrg1 in development and maintenance of the myelin sheath. Neurobiol Dis 2011;42:368-80.

12 Zheng Y, Wang LS, Xia L, et al. NDRG1 is down-regulated in the early apoptotic event induced by camptothecin analogs: the potential role in proteolytic activation of PKC delta and apoptosis. Proteomics 2009;9:2064-75.

13 Bandyopadhyay S, Wang $Y$, Zhan R, et al. The tumor metastasis suppressor gene Drg-1 down-regulates the expression of activating transcription factor 3 in prostate cancer. Cancer Res 2006;66:11983-90.

14 Matsugaki T, Zenmyo M, Hiraoka K, et al. N-myc downstream-regulated gene 1/Cap43 expression promotes cell differentiation of human osteosarcoma cells. Oncol Rep 24:721-5.

15 Chua MS, Sun H, Cheung ST, et al. Overexpression of NDRG1 is an indicator of poor prognosis in hepatocellular carcinoma. Mod Pathol 2007;20:76-83.

16 Cowling VH, Chandriani S, Whitfield ML, et al. A conserved Myc protein domain, MBIV, regulates DNA binding, apoptosis, transformation, and G2 arrest. Mol Cell Biol 2006;26:4226-39.

17 Herbst A, Hemann MT, Tworkowski KA, et al. A conserved element in Myc that negatively regulates its proapoptotic activity. EMBO Rep 2005;6:177-83.

18 Oster SK, Ho CS, Soucie EL, et al. The myc oncogene: MarvelouslY Complex. Adv Cancer Res 2002;84:81-154.
19 Bandyopadhyay S, Pai SK, Gross SC, et al. The Drg-1 gene suppresses tumor metastasis in prostate cancer. Cancer Res 2003;63:1731-6.

20 Toffoli S, Delaive E, Dieu M, et al. NDRG1 and CRK-1/II are regulators of endothelial cell migration under intermittent hypoxia. Angiogenesis 2009;12:339-54.

21 Cangul H. Hypoxia upregulates the expression of the NDRG1 gene leading to its overexpression in various human cancers. BMC Genet 2004;5:27.

22 Lachat $\mathrm{P}$, Shaw $\mathrm{P}$, Gebhard $\mathrm{S}$, et al. Expression of NDRG1, a differentiation-related gene, in human tissues. Histochem Cell Biol 2002;118:399-408.

23 Chen $S$, Han YH, Zheng Y, et al. NDRG1 contributes to retinoic acid-induced differentiation of leukemic cells. Leuk Res 2009;33:1108-13.

24 Nishio S, Ushijima K, Tsuda N, et al. Cap43/NDRG1/Drg-1 is a molecular target for angiogenesis and a prognostic indicator in cervical adenocarcinoma. Cancer Lett 2008;264:36-43.

25 Nishie A, Masuda K, Otsubo M, et al. High expression of the Cap43 gene in infiltrating macrophages of human renal cell carcinomas. Clin Cancer Res 2001;7:2145-51.

26 Kovacevic Z, Chikhani S, Lui GY, et al. The iron-regulated metastasis suppressor NDRG1 targets NEDD4L, PTEN, and SMAD4 and inhibits the PI3K and Ras signaling pathways. Antioxid Redox Signal 2013;18:874-87.

27 Akiba J, Ogasawara S, Kawahara A, et al. N-myc downstream regulated gene 1 (NDRG1)/Cap43 enhances portal vein invasion and intrahepatic metastasis in human hepatocellular carcinoma. Oncol Rep 2008;20:1329-35.

28 Jung EU, Yoon JH, Lee YJ, et al. Hypoxia and retinoic acid-inducible NDRG1 expression is responsible for doxorubicin and retinoic acid resistance in hepatocellular carcinoma cells. Cancer Lett 2010;298:9-15.

29 Li J, Kretzner L. The growth-inhibitory Ndrg1 gene is a Myc negative target in human neuroblastomas and other cell types with overexpressed N- or c-myc. Mol Cell Biochem 2003;250:91-105.

30 Liu $W$, Xing $F$, liizumi-Gairani $M$, et al. N-myc downstream regulated gene 1 modulates Wnt-beta-catenin signalling and pleiotropically suppresses metastasis. EMBO Mol Med 2012;4:93-108.

31 Akiba J, Murakami Y, Noda M, et al. N-myc downstream regulated gene1/Cap43 overexpression suppresses tumor growth by hepatic cancer cells through cell cycle arrest at the G0/G1 phase. Cancer Lett 2011;310:25-34.

32 Chang JT, Wang HM, Chang KW, et al. Identification of differentially expressed genes in oral squamous cell carcinoma (OSCC): overexpression of NPM, CDK1 and NDRG1 and underexpression of CHES1. Int I Cancer 2005;114:942-9.

33 Kovacevic $Z$, Sivagurunathan $\mathrm{S}$, Mangs $\mathrm{H}$, et al. The metastasis suppressor, N-myc downstream regulated gene 1 (NDRG1), upregulates p21 via p53-independent mechanisms. Carcinogenesis 2011;32:732-40.

34 Kim KT, Ongusaha PP, Hong YK, et al. Function of Drg1/Rit42 in p53-dependent mitotic spindle checkpoint. J Biol Chem 2004;279:38597-602.

35 Fotovati A, Fujii T, Yamaguchi M, et al. 17Beta-estradiol induces down-regulation of Cap43/NDRG1/Drg-1, a putative differentiation-related and metastasis suppressor gene, in human breast cancer cells. Clin Cancer Res 2006;12:3010-18.

36 McCaig C, Potter L, Abramczyk O, et al. Phosphorylation of NDRG1 is temporally and spatially controlled during the cell cycle. Biochem Biophys Res Commun 2011;411:227-34.

37 Chen Z, Zhang D, Yue F, et al. The iron chelators Dp44mT and DFO inhibit TGF-beta-induced epithelial-mesenchymal transition via up-regulation of N-Myc downstream-regulated gene 1 (NDRG1). J Biol Chem 2012;287:17016-28.

38 Stein S, Thomas EK, Herzog B 2nd, et al. NDRG1 is necessary for p53-dependent apoptosis. J Biol Chem 2004;279:48930-40.

39 Zhang AH, Rao JN, Zou T, et al. p53-dependent NDRG1 expression induces inhibition of intestinal epithelial cell proliferation but not apoptosis after polyamine depletion. Am J Physiol Cell Physiol 2007;293:C379-89.

40 Lane DJ, Saletta F, Rahmanto Y Suryo, et al. N-myc downstream regulated 1 (NDRG1) is regulated by eukaryotic initiation factor $3 a$ (elF3a) during cellular stress caused by iron depletion. PLoS One 2013;8:e57273.

41 Lluis JM, Buricchi F, Chiarugi $P$, et al. Dual role of mitochondrial reactive oxygen species in hypoxia signaling: activation of nuclear factor-\{kappa\}B via c-SRC and oxidant-dependent cell death. Cancer Res 2007;67:7368-77.

42 Zhang $\mathrm{P}$, Tchou-Wong KM, Costa M. Egr-1 mediates hypoxia-inducible transcription of the NDRG1 gene through an overlapping Egr-1/Sp1 binding site in the promoter. Cancer Res 2007;67:9125-33.

43 Fruehauf JP, Meyskens FL Jr. Reactive oxygen species: a breath of life or death? Clin Cancer Res 2007;13:789-94.

44 Mazure NM, Pouyssegur J. Hypoxia-induced autophagy: cell death or cell survival? Curr Opin Cell Biol 2010;22:177-80.

45 Caruso RP, Levinson B, Melamed J, et al. Altered N-myc downstream-regulated gene 1 protein expression in African-American compared with caucasian prostate cancer patients. Clin Cancer Res 2004;10:222-7.

46 Le NT, Richardson DR. Iron chelators with high antiproliferative activity up-regulate the expression of a growth inhibitory and metastasis suppressor gene: a link between iron metabolism and proliferation. Blood 2004;104:2967-75.

47 Salnikow K, Su W, Blagosklonny MV, et al. Carcinogenic metals induce hypoxia-inducible factor-stimulated transcription by reactive oxygen species-independent mechanism. Cancer Res 2000;60:3375-8. 
48 Park $H$, Adams MA, Lachat $P$, et al. Hypoxia induces the expression of a 43-kDa protein (PROXY-1) in normal and malignant cells. Biochem Biophys Res Commun 2000;276:321-8.

49 Kedersha N, Anderson P. Stress granules: sites of mRNA triage that regulate mRNA stability and translatability. Biochem Soc Trans 2002:30:963-9.

50 Anderson P, Kedersha N. Stress granules: the Tao of RNA triage. Trends Biochem Sci 2008;33:141-50.

51 Bandyopadhyay S, Pai SK, Hirota S, et al. Role of the putative tumor metastasis suppressor gene Drg-1 in breast cancer progression. Oncogene 2004;23:5675-81.

52 Li X, Sakamoto K, Takahashi Y, et al. Overexpression of Cap43 gene in supraglottic laryngeal squamous cell carcinoma. J Laryngol Otol 2009;123(Suppl 31):11-17.

53 Redmond KL, Crawford NT, Farmer $\mathrm{H}$, et al. T-box 2 represses NDRG1 through an EGR1-dependent mechanism to drive the proliferation of breast cancer cells. Oncogene 2010;29:3252-62.

54 Inagaki Y, Tang W, Xu HL, et al. Localization of N-myc downstream-regulated gene 1 in gastric cancer tissue. Dig Liver Dis 2009:41:96-103.

55 Kovacevic Z, Chikhani S, Lovejoy DB, et al. Novel thiosemicarbazone iron chelators induce up-regulation and phosphorylation of the metastasis suppressor N-myc down-stream regulated gene 1: a new strategy for the treatment of pancreatic cancer. Mol Pharmacol 2011;80:598-609.

56 Gerhard R, Nonogaki S, Fregnani JH, et al. NDRG1 protein overexpression in malignant thyroid neoplasms. Clinics (Sao Paulo) 2010;65:757-62.

57 Shimono A, Okuda T, Kondoh H. N-myc-dependent repression of ndr1, a gene identified by direct subtraction of whole mouse embryo cDNAs between wild type and N-myc mutant. Mech Dev 1999;83:39-52.

58 Chung LC, Tsui KH, Feng TH, et al. L-Mimosine blocks cell proliferation via upregulation of $\mathrm{B}$-cell translocation gene 2 and $\mathrm{N}$-myc downstream regulated gene 1 in prostate carcinoma cells. Am J Physiol Cell Physiol 2012;302:C676-85.

59 Song $Y$, Oda $Y$, Hori $M$, et al. N-myc downstream regulated gene- $1 /$ Cap43 may play an important role in malignant progression of prostate cancer, in its close association with E-cadherin. Hum Pathol 2010;41:214-22.

60 Li Q, Chen H. Transcriptional silencing of N-Myc downstream-regulated gene 1 (NDRG1) in metastatic colon cancer cell line SW620. Clin Exp Metastasis 2011:28:127-35.

61 Maruyama Y, Ono M, Kawahara A, et al. Tumor growth suppression in pancreatic cancer by a putative metastasis suppressor gene Cap43/NDRG1/Drg-1 through modulation of angiogenesis. Cancer Res 2006;66:6233-42.

62 Kurdistani SK, Arizti P, Reimer CL, et al. Inhibition of tumor cell growth by RTP/rit42 and its responsiveness to p53 and DNA damage. Cancer Res 1998;58:4439-44.

63 Guan RJ, Ford HL, Fu Y, et al. Drg-1 as a differentiation-related, putative metastatic suppressor gene in human colon cancer. Cancer Res 2000;60:749-55.

64 Lai LC, Su YY, Chen KC, et al. Down-regulation of NDRG1 promotes migration of cancer cells during reoxygenation. PLoS One 2011;6:e24375.

65 Jiang $K$, Shen $Z$, Ye Y, et al. A novel molecular marker for early detection and evaluating prognosis of gastric cancer: $\mathrm{N}$-myc downstream regulated gene-1 (NDRG1). Scand J Gastroenterol 2010;45:898-908.

66 Strzelczyk B, Szulc A, Rzepko R, et al. Identification of high-risk stage II colorectal tumors by combined analysis of the NDRG1 gene expression and the depth of tumor invasion. Ann Surg Oncol 2009;16:1287-94.

67 Liu W, liizumi-Gairani M, Okuda H, et al. KAl1 gene is engaged in NDRG1 gene-mediated metastasis suppression through the ATF3-NFkappaB complex in human prostate cancer. J Biol Chem 2011:286:18949-59.
68 Dominguez R, Holmes KC. Actin structure and function. Annu Rev Biophys 2011;40:169-86.

69 Chaturvedi LS, Marsh HM, Basson MD. Role of RhoA and its effectors ROCK and mDia1 in the modulation of deformation-induced FAK, ERK, p38, and MLC motogenic signals in human Caco-2 intestinal epithelial cells. Am J Physiol Cell Physiol 2011;301:C1224-38.

70 Sun J, Zhang D, Zheng Y, et al. Targeting the metastasis suppressor, NDRG1, using novel iron chelators: regulation of stress fiber-mediated tumor cell migration via modulation of the ROCK1/pMLC2 signaling pathway. Mol Pharmacol 2013:83:454-69.

71 Liu YL, Bai WT, Luo W, et al. Downregulation of NDRG1 promotes invasion of human gastric cancer AGS cells through MMP-2. Tumour Biol 2011;32:99-105

72 Hosoi F, Izumi H, Kawahara A, et al. N-myc downstream regulated gene 1/Cap43 suppresses tumor growth and angiogenesis of pancreatic cancer through attenuation of inhibitor of kappaB kinase beta expression. Cancer Res 2009;69:4983-91.

73 Weiner LM, Lotze MT. Tumor-cell death, autophagy, and immunity. N Eng/ J Med 2012:366:1156-8.

74 Angst E, Dawson DW, Stroka D, et al. N-myc downstream regulated gene-1 expression correlates with reduced pancreatic cancer growth and increased apoptosis in vitro and in vivo. Surgery 2011;149:614-24.

75 Whitnall $\mathrm{M}$, Howard J, Ponka $\mathrm{P}$, et al. A class of iron chelators with a wide spectrum of potent antitumor activity that overcomes resistance to chemotherapeutics. Proc Natnl Acad Sci 2006;103:14901-6.

76 Yu Y, Rahmanto YS, Richardson D. Bp44mT: an orally active iron chelator of the thiosemicarbazone class with potent anti-tumour efficacy. Brit J Pharmacol 2012;165:148-66.

77 Lovejoy DB, Sharp DM, Seebacher N, et al. Novel second-generation di-2-pyridylketone thiosemicarbazones show synergism with standard chemotherapeutics and demonstrate potent activity against lung cancer xenografts after oral and intravenous administration in vivo. J Med Chem 2012;55:7230-44.

78 Yuan J, Lovejoy DB, Richardson DR. Novel di-2-pyridyl-derived iron chelators with marked and selective antitumor activity: in vitro and in vivo assessment. Blood 2004:104:1450-8.

79 Richardson DR, Sharpe PC, Lovejoy DB, et al. Dipyridyl thiosemicarbazone chelators with potent and selective antitumor activity form iron complexes with redox activity. J Med Chem 2006;49:6510-21.

80 Lovejoy DB, Jansson PJ, Brunk UT, et al. Antitumor activity of metal-chelating compound Dp44mT is mediated by formation of a redox-active copper complex that accumulates in lysosomes. Cancer Res 2011;71:5871-80.

81 Kalinowski DS, Richardson DR. The evolution of iron chelators for the treatment of iron overload disease and cancer. Pharmacol Rev 2005;57:547-83.

82 Richardson DR, Baker $\mathrm{E}$. The uptake of iron and transferrin by the human malignant melanoma cell. Biochimica et biophysica acta 1990;1053:1-12.

83 Richardson DR, Baker E. Intermediate steps in cellular iron uptake from transferrin. Detection of a cytoplasmic pool of iron, free of transferrin. J Biol Chem 1992;267:21384-9.

$84 \mathrm{Yu}$ Y, Kovacevic Z, Richardson DR. Tuning cell cycle regulation with an iron key. Cell Cycle 2007;6:1982-94.

85 Coombs GS, Schmitt AA, Canning CA, et al. Modulation of Wnt/beta-catenin signaling and proliferation by a ferrous iron chelator with therapeutic efficacy in genetically engineered mouse models of cancer. Oncogene 2012;31:213-25. 